\title{
Altered lipid subfraction profile and impaired antioxidant defense of high-density lipoprotein in Smith-Lemli-Opitz syndrome
}

\author{
Hajnalka Lőrincz', Mariann Harangi', Anna V. Oláh², Gabriella P. Szabó³ , Péter Fülöp' ', Sándor Somodi', György Paragh' and \\ Ildikó Seres ${ }^{1}$
}

BACKGROUND: Smith-Lemli-Opitz syndrome (SLOS) is a rare disease caused by biallelic mutation in the 7-dehydrocholesterol (7DHC) reductase gene. High oxidizability of 7DHC and the appearance of small-sized low-density lipoprotein $(\mathrm{LDL})$ subfractions indicate increased endogenous oxidative stress that is counterbalanced by natural antioxidant defense mechanisms including the high-density lipoprotein (HDL)associated paraoxonase-1 (PON1) enzyme. PON1 prevents lipoproteins from oxidative modifications; however, PON1 activity and the distribution of lipoprotein subfractions have not been studied in SLOS.

METHODS: 7DHC levels and PON1 arylesterase activities were measured spectrophotometrically in 11 SLOS patients and 10 healthy children. Lipoprotein subfractions were detected by polyacrylamide gel electrophoresis.

RESULTS: Compared to controls, there was a shift towards the small-dense LDL subfraction and the large HDL subfraction in SLOS. PON1 arylesterase activity was significantly decreased in SLOS patients and correlated negatively with the proportion of small-dense LDL subfraction and the proportion of large HDL subfraction. Significant positive correlations were detected between PON1 arylesterase activity and the ratios of intermediate and small HDL subfractions.

CONCLUSIONS: Decreased PON1 activity and the deleterious shift in the distribution of lipoprotein subfractions may contribute to the impaired antioxidant status observed in SLOS. Monitoring of serum PON1 arylesterase activity may be a complementary biomarker in SLOS.

\section{INTRODUCTION}

Cholesterol is an important structural component of cell membranes and serves as a precursor of steroid hormones and bile acids. It is a major constituent of the myelin formed in the brain, spinal fluid and in the peripheral nervous system. From all organs, brain is the richest in cholesterol, containing $\sim 20 \%$ of the whole body cholesterol (1). Cholesterol is synthesized from lanosterol by a series of oxidations, reductions and demethylations (2); therefore, it is not surprising that inherited defects of cholesterol biosynthesis may lead to numerous diseases.

Smith-Lemli-Opitz syndrome (SLOS, OMIM 270400) is a rare autosomal recessive genetic disease originating from biallelic mutations in the gene encoding $3 \beta$-hydroxysterol- $\Delta^{7}$ reductase (DHCR7, EC 1.3.1.21), which is located on chromosome 11q13. This genetic defect is manifested as a large and variable spectrum of phenotypes, including multiple congenital malformations, microcephaly, neurological defects, photosensitivity, syndactyly of the second and third toes, growth and mental retardation, and behavioral problems $(3,4)$. For phenotypic characterization, the modified Bialer scoring system of Kelley and Hennekam has been used weighting embryologically separate organ systems equally (5). DHCR7 enzyme catalyzes the reduction of 7-dehydrocholesterol (7DHC) to cholesterol in the last step of cholesterol biosynthesis pathway (6). SLOS patients exhibit extremely high levels of 7DHC and reduced levels of cholesterol in the tissues, plasma and other fluids. In accordance with the results of Tint et al. (7), we previously reported that both the initial value of serum cholesterol level and the cholesterol/7DHC ratio had an important prognostic value in the severity, development and life expectancy of SLOS children (8). To date, routine therapy has been cholesterol supplementation in SLOS; while combined cholesterol-simvastatin therapy $(9,10)$ or cholesterol-antioxidant treatment might also be effective in SLOS patients (11). However, as it was recently reviewed, simvastatin is not recommended for SLOS treatment due to its potential side effects (12). High oxidizability of 7DHC has been reported to enhance endogenous oxidative stress in cell and animal models of SLOS (13) and in several human studies (14). Additionally, inactivation of the endogenous antioxidant defense mechanisms may also contribute to increased oxidative stress. Oxidation of the predominantly smaller and denser low-density lipoprotein (LDL) particles play a key role in this process (15). Human paraoxonase-1 (PON1) is a high-density lipoprotein (HDL)associated enzyme with significant antioxidant properties preventing lipoproteins from oxidative modification (16). PON1 
activity was found to diminish in several human diseases characterized by enhanced oxidative stress, including coronary artery disease, diabetes mellitus, hyperlipidemia, obesity, and several autoimmune diseases (17-20).

As it was demonstrated recently, high 7DHC level accelerates the formation of oxysterols in SLOS resulting in increased cellular susceptibility for oxidative stress $(21,22)$. To date, however, there is no data on the distribution of lipoprotein subfractions in SLOS, while information about the antioxidant PON1 activity is also lacking in these patients. Therefore, we aimed to study the distributions of LDL and HDL subfractions in SLOS patients and compared their data to healthy children. We also examined PON1 activities in these groups and intended to clarify possible associations between PON1 antioxidant status and various lipoprotein subfractions.

\section{RESULTS}

Clinical and laboratory characteristics of study participants are summarized in Table 1. Mean SLOS clinical severity score was $30.9 \pm 15.1$; one child with severe disease (clinical severity score $>50$ ) died as a newborn, seven patients exhibited typical symptoms (clinical severity score 20-50), and three patients had mild features of SLOS group (clinical severity score $<20$ ). 7DHC level in SLOS patients was $205(85-274) \mathrm{mg} / \mathrm{l}$, while 7DHC levels were below the limit of detection in healthy children $(<0.15 \mathrm{mg} / \mathrm{l})$. Mean total cholesterol, LDL-C and HDL-C levels were significantly lower in patients with SLOS

Table 1 Clinical and laboratory characteristics of the study participants

\begin{tabular}{|c|c|c|c|}
\hline & $\operatorname{SLOS}(n=11)$ & Control $(n=10)$ & $P$ \\
\hline Gender & 4 girls $/ 7$ boys & 5 girls $/ 5$ boys & \\
\hline Age (years) & $5.71 \pm 6.93$ & $5.36 \pm 6.02$ & n.s. \\
\hline Clinical severity score & $30.9 \pm 15.1$ & & \\
\hline $\begin{array}{l}\text { 7-Dehydrocholesterol } \\
(\mathrm{mg} / \mathrm{l})\end{array}$ & $205(85-274)$ & n.d. & \\
\hline $\begin{array}{l}\text { Total-Cholesterol } \\
(\mathrm{mmol} / \mathrm{l})\end{array}$ & $1.73 \pm 0.9$ & $3.52 \pm 0.63$ & $<0.0001$ \\
\hline LDL-C (mmol/l) & $0.9 \pm 0.4$ & $1.88 \pm 0.46$ & $<0.0001$ \\
\hline Large LDL (\%) & $16.9(14.5-22.4)$ & $26.1(23.8-30.4)$ & $<0.001$ \\
\hline Small-dense LDL (\%) & $10.2(2.3-13.3)$ & $0.8(0.0-2.7)$ & $<0.01$ \\
\hline Mean LDL size (nm) & $25.7 \pm 0.83$ & $27.16 \pm 0.32$ & $<0.001$ \\
\hline \multicolumn{4}{|l|}{ LDL subfraction pattern } \\
\hline Normal $(n)$ & 3 & 8 & \\
\hline Intermediate $(n)$ & 1 & 2 & \\
\hline Abnormal (n) & 7 & 0 & \\
\hline $\mathrm{HDL}-\mathrm{C}(\mathrm{mmol} / \mathrm{l})$ & $0.55 \pm 0.38$ & $1.04 \pm 0.27$ & $<0.01$ \\
\hline Large HDL (\%) & $73.5(47.4-77.2)$ & $34.5(31.6-41.8)$ & $<0.001$ \\
\hline Intermediate HDL (\%) & $19.8(18.9-39.6)$ & $49.5(44.3-50.5)$ & $<0.001$ \\
\hline Small HDL (\%) & $7.1(2.3-11.0)$ & $16.0(13.9-18.5)$ & $<0.01$ \\
\hline
\end{tabular}

Values are presented as mean \pm SD or median (lower quartile to upper quartile).

LDL-C, low-density lipoprotein cholesterol; HDL-C, high-density lipoprotein cholesterol; n.s., nonsignificant; n.d., nondetectable, $<0.15$ mg/l; SLOS, Smith-Lemli-Opitz syndrome.
( $P<0.0001, P<0.0001$, and $P<0.01$; respectively) compared to healthy children. A strong, significant negative correlation was detected between the clinical severity score and HDL-C levels ( $r=-0.804, P=0.003$ ) (Figure 1a).

Compared to controls, the proportion of large LDL subfraction was significantly lower $(P<0.001)$, while the proportion of small-sized, dense LDL subfraction was significantly higher $(P<0.01)$ in SLOS patients (Table 1). Furthermore, the mean LDL size was significantly lower in patients with SLOS than in healthy children $(P<0.001)$. These data are in accordance with the LDL subfraction pattern in these patients: seven SLOS patients represented an abnormal LDL pattern; one child had intermediate and only three children had normal LDL subfraction pattern. In contrast, none of the control children displayed abnormal LDL subfraction pattern, two of them were found to have an intermediate pattern, while the remaining eight children represented normal LDL subfraction patterns. Figure 2 demonstrates an abnormal subfraction pattern (mean LDL size: $25.34 \mathrm{~nm}$ ) in a typical SLOS patient

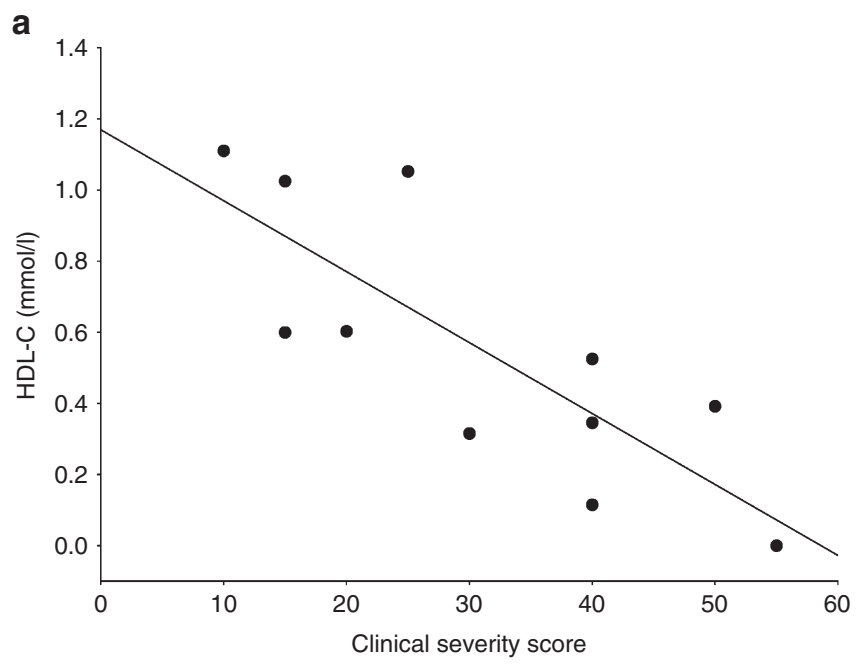

b

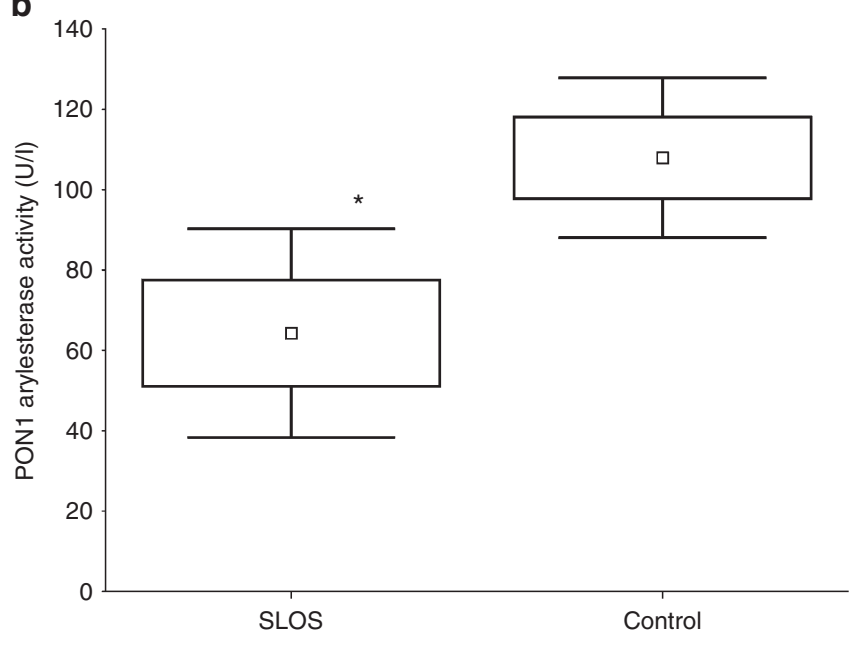

Figure 1. Correlation between clinical severity scores and HDL-C levels in SLOS patients $(n=11 ; r=-0.804 ; P=0.003)(\mathbf{a})$; Serum PON1 arylesterase activity in SLOS and healthy control children ( ${ }^{*} P=0.022 ; \square$ mean; box: mean \pm SD; whiskers: mean \pm 1.96 SD) (b). 


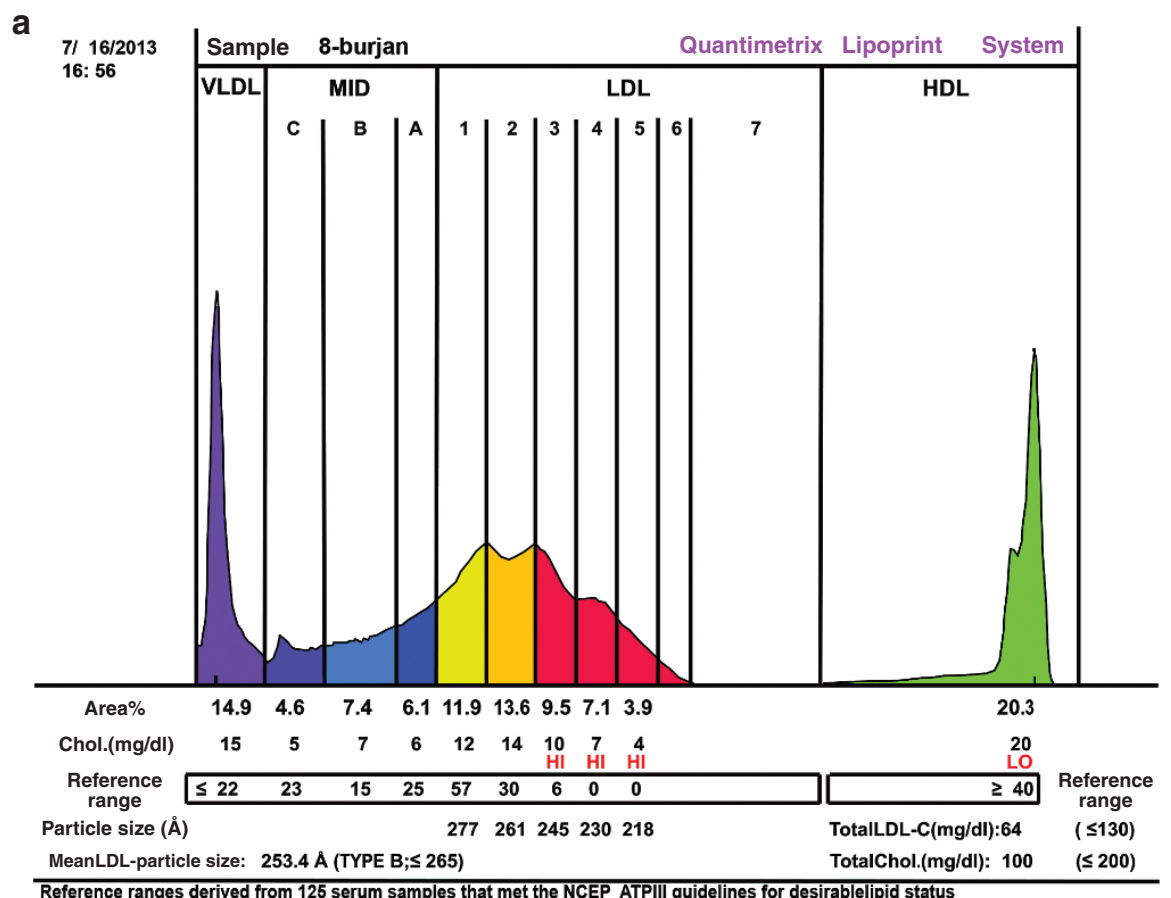

Reference ranges derived from 125 serum samples that met the NCEP ATPIII guidelines for desirablelipid status
LDL-C is comprised of the sum of cholesterol in Mid bands $C$ through $A$ as well as all the subfractions

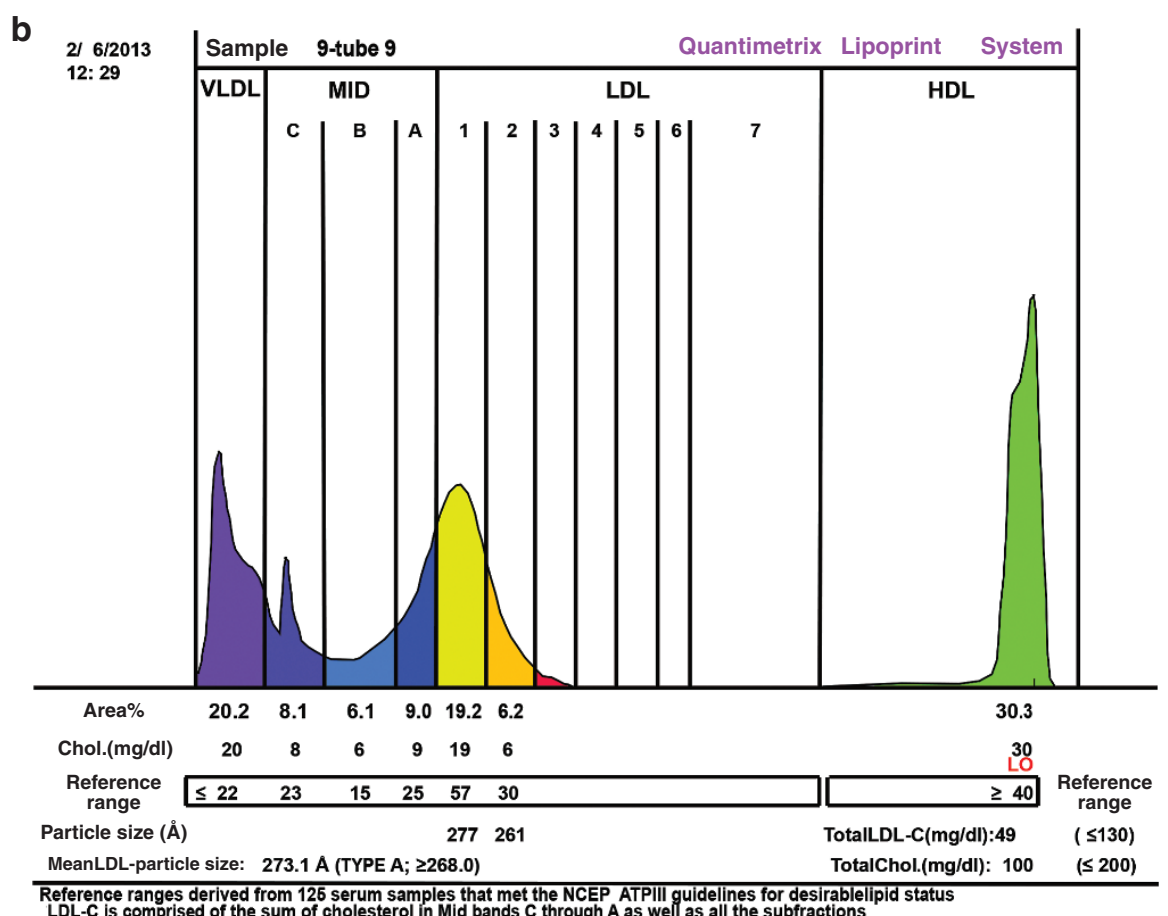

Figure 2. LDL subfraction profile (a) in a typical SLOS patient and (b) in a healthy child.

(Figure 2a) and a normal LDL subfraction pattern (mean LDL size: $27.31 \mathrm{~nm}$ ) in a healthy child (Figure $2 \mathbf{b}$ ). Densitometric scanning of LDL subfractions identified an SLOS patient with predominantly smaller and denser LDL subfractions (shown in red in Figure 2a) indicating an increased susceptibility for enhanced oxidative stress in SLOS. On the other hand, Figure $2 \mathbf{b}$ is a representative sample of lipoprotein subfraction pattern of a healthy child typically with rather larger and less dense LDL subfraction pattern (shown in yellow).
We also detected a shift towards the larger and less dense HDL subfractions in children with SLOS (Table 1). The proportion of the large HDL subfractions was significantly higher $(P<0.001)$, while proportions of intermediate and small HDL subfractions were significantly lower in SLOS children compared to healthy controls $(P<0.001$ and $P<0.01$, respectively). In Figure 3, we also demonstrate a densitometric scan of HDL subfractions in a patient with typical SLOS (Figure 3a) representing the dominance of large HDL subfractions and the 
a

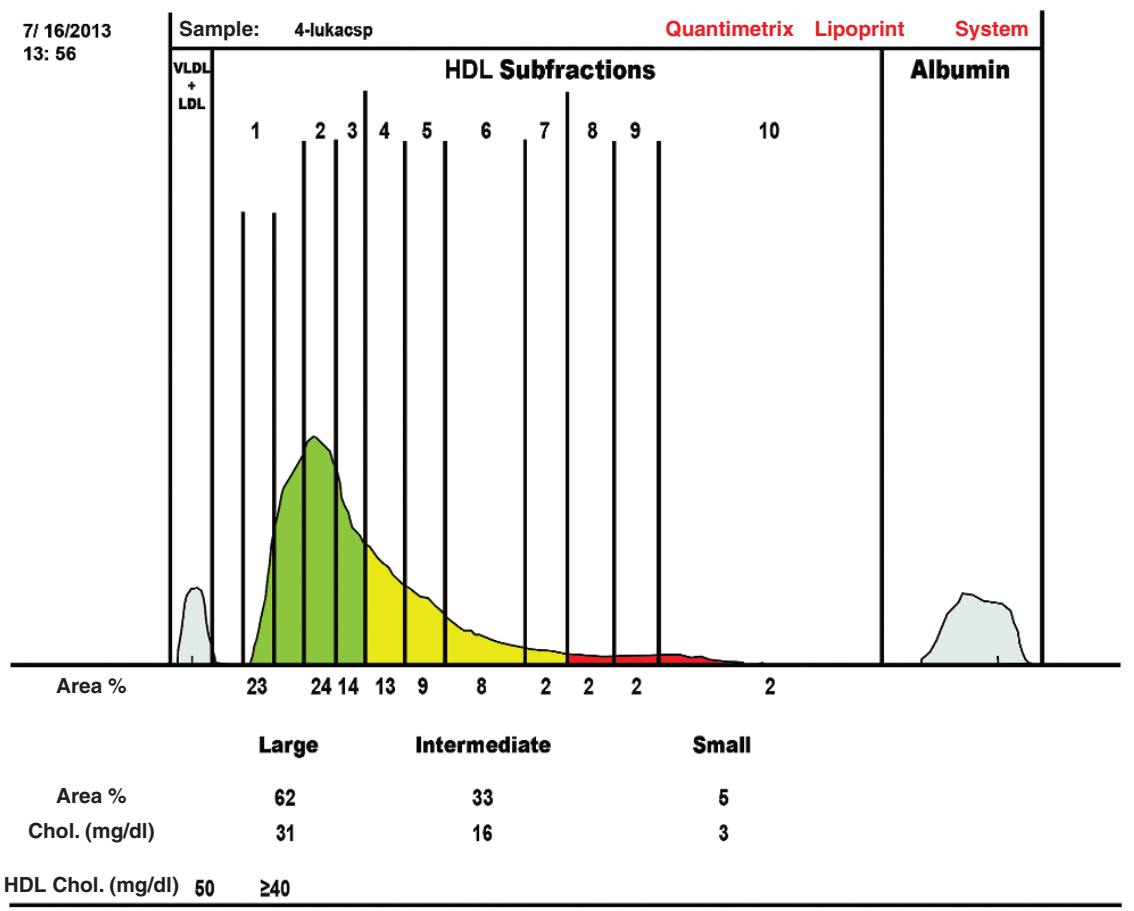

b

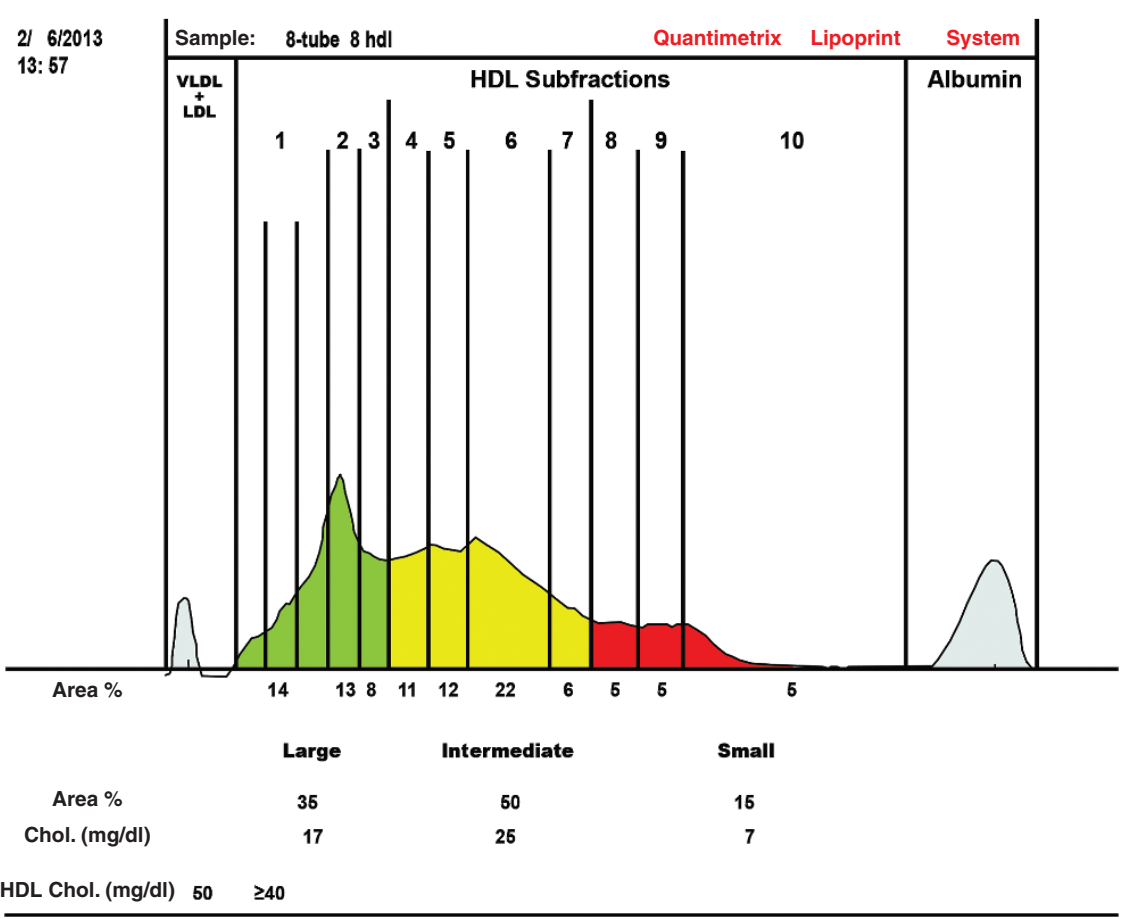

Figure 3. HDL subfraction profile (a) in a typical SLOS patient and (b) in a healthy child.

decreased prevalence of intermediate and small HDL subfractions compared to a healthy control child (Figure 3b).

Serum PON1 paraoxonase activity was lower in the SLOS group compared to controls, SLOS: 37.05 (9.98-93.22) U/L vs. controls: 72.85 (42.04-206.93) U/L; $P=0.069$; although the difference was not significant between the two groups. In turn, serum PON1 arylesterase activity was found to be significantly decreased in SLOS children compared to controls
(SLOS: $64.30 \pm 52.69 \mathrm{U} / \mathrm{L}$ vs. controls: $107.93 \pm 26.83 \mathrm{U} / \mathrm{L} ; P=$ 0.022) (Figure 1b).

Examining the correlations between serum PON1 arylesterase activity and lipoprotein parameters (Table 2), we detected a significant positive correlation between PON1 arylesterase activity and total cholesterol level $(r=0.543, P$ $=0.045)$. In addition, there were positive, although not significant trends of associations between PON1 arylesterase 
Table 2 Correlations between serum PON1 arylesterase activity and lipoprotein parameters in all studied participants

\begin{tabular}{lcc}
\hline Variables & $r$ & $P$ \\
\hline Total-cholesterol (mmol/l) & 0.543 & 0.045 \\
LDL-C (mmol/l) & 0.533 & n.s. \\
Large LDL (\%) & 0.349 & n.s. \\
Small-dense LDL (\%) & -0.578 & 0.031 \\
Mean LDL size (nm) & 0.610 & 0.021 \\
HDL-C (mmol/l) & 0.457 & n.s. \\
Large HDL (\%) & -0.798 & 0.001 \\
Intermediate HDL (\%) & 0.652 & 0.012 \\
Small HDL (\%) & 0.663 & 0.010 \\
\hline LDL-C, low-density lipoprotein cholesterol; HDL-C, high-density lipoprotein cholesterol; \\
n.s. nonsignificant.
\end{tabular}

activities and LDL-C concentrations and the proportions of large LDL subfractions, respectively. In turn, a significant negative correlation was found between PON1 arylesterase activity and the proportion of small-dense LDL subfraction $(r=-0.578, P=0.031)$. Additionally, PON1 arylesterase activity correlated positively with mean LDL size $(r=0.610$, $P=0.021$ ).

Although there was no significant association between PON1 arylesterase activity and HDL-C levels; PON1 arylesterase activity correlated negatively with the proportion of large HDL subfraction $(r=-0.798, P=0.001)$. In contrast, significant positive correlations were found between PON1 arylesterase activities and the proportions of intermediate and small HDL subfractions $(r=0.652, P=0.012$ and $r=0.663$, $P=0.010)$, respectively.

\section{DISCUSSION}

According to our knowledge, this is first report on the distribution of LDL and HDL subfractions and the status of HDLassociated antioxidant PON1 activity in SLOS patients. Based upon the lipoprotein subfraction analyses, we detected a shift towards the small-dense LDL subfraction and the dominance of the large-sized HDL subfraction, which indicate disturbed cholesterol biosynthesis in SLOS. Decreased PON1 arylesterase activity and elevated levels of 7DHC were also demonstrated in these children, revealing enhanced endogenous oxidative stress and impaired antioxidant status.

Previous studies found altered lipoprotein parameters and impaired cholesterol biosynthesis in SLOS $(23,24)$. Also, initial cholesterol concentrations were found to be fundamental in determining severity and life expectancy in SLOS $(7,8)$. Corroborating previous data, significantly decreased total cholesterol, LDL-C and HDL-C levels were measured in our patients and we found a significant negative correlation between HDL-C level and the clinical severity score, emphasizing the putative role of HDL in the pathomechanism of SLOS.

LDL and HDL are heterogeneous particles consisting multiple lipoprotein subpopulations that vary in protein and lipid components, physicochemical properties, particle size and density (25). Although lipoprotein levels are essential in the prognosis of SLOS, the distributions of LDL and HDL subfractions have not been studied yet. Compared to healthy infants, we found an unexpectedly higher proportion of small-dense LDL subfraction and lower mean LDL size in children with SLOS. The prevalence of abnormal LDL subfraction pattern was $63 \%$ in SLOS, whereas none of the healthy children displayed LDL abnormality. The latter data are in congruence with the results of the STRIP Study indicating a $1-4 \%$ prevalence of the small-dense LDL subfraction in healthy children (26). Since the predominantly smaller and denser LDL subfractions are extremely prone to undergo oxidative modification (15), their dominance might indicate the presence of oxidative stress in SLOS; however, further studies are needed to clarify such association in this disease.

HDL subpopulations play various roles in lipid metabolism, as large-sized HDL subfractions are primarily involved in reverse cholesterol transport-a mechanism by which HDL takes up cholesterol in the peripheral tissues and transports it to the liver; while small-sized HDL subfractions rather exert anti-inflammatory effects protecting LDL from oxidation $(27,28)$. In this study, we observed altered distribution of HDL subfractions in SLOS patients who were characterized by a significantly higher proportion of large-sized HDL subfraction and lower proportions of intermediate and smallsized HDL subfractions. Based upon these results, one may hypothesize that altered distribution of HDL subfractions may reflect enhanced reverse cholesterol transport compensating for extremely low cholesterol levels found routinely in SLOS. HDL-associated PON1 is widely recognized as one of the major antioxidant enzymes of HDL and localized primarily on small-sized HDL subfractions (29,30). Diminished PON1 activity was reported in several disease entities involving altered redox balance and increased oxidative stress (31). Therefore, lower proportion of small-sized HDL subfractions and decreased PON1 arylesterase activity in SLOS patients might be related to their impaired synthesis in the liver and they probably indicate a reduced antioxidant capacity.

It must be noted, that PON1 possesses various enzyme activities with different substrate specificities including paraoxonase and arylesterase activities. PON1 paraoxonase activity is determined by a Q192R polymorphism in the coding region and shows a trimodal distribution; therefore, PON1 paraoxonase activity has a large inter-individual variation (up to $15-40$ times) among the general population (31). In contrast, PON1 arylesterase activity is not affected by this polymorphism; therefore, this activity is distributed unimodally and reflects protein concentration and antioxidant capacity of PON1 $(32,33)$. Compared to controls, we found a marked but not significant reduction of serum PON1 paraoxonase activity in children with SLOS. Presumably due to the relatively small sample size and the trimodal distribution of the PON1 paraoxonase activity, we did not detect a difference in this variable between the studied groups. In turn, we demonstrated a significant negative correlation between the proportion of 
oxidizable small-dense LDL subfraction and the antioxidant PON1 arylesterase activity. The proportion of large HDL subfraction correlated negatively, while the proportions of intermediate and small HDL subfractions correlated positively with PON1 arylesterase activity. These correlations support previous observations that small-sized HDL subfraction may have the highest PON1 activity (34). Thus, one might conclude that decreased PON1 arylesterase activity and altered lipoprotein subfractions may serve as novel biomarkers in SLOS.

In line with decreased PON1 arylesterase activity, we also found markedly increased levels of 7DHC further enhancing oxidative stress in SLOS. 7HDC is highly disposed to react with molecular oxygen, producing over a dozen different oxysterols, such as $3 \beta, 5 \alpha$-dihydroxycholest-7-en-6-one (DHCEO) in vitro and in vivo $(21,22,35)$. Since $7 \mathrm{DHC}$ and $7 \mathrm{DHC}$-derived oxysterols are potent pro-oxidant agents, these molecules may also contribute to enhanced lipid peroxidation and protein degradation resulting in reduced antioxidant capacity and PON1 activity.

\section{Conclusion}

Decreased PON1 arylesterase activity and an unfavorable shift in the distribution of lipoprotein subfractions may contribute to impaired antioxidant status in SLOS. Monitoring of serum PON1 arylesterase activity may serve as a useful complementary biomarker in these patients. Further studies are needed to determine the clinical benefits of PON1 arylesterase activity measurement in SLOS.

\section{METHODS}

\section{Study Population}

Eleven children with clinical and biochemical diagnosis of SLOS (aged between $0.1-20 \mathrm{y}$, four girls and seven boys) and 10 healthy control children (age of 0.3-19y, five girls and five boys) were enrolled in our study. Besides the presence of characteristic clinical symptoms, the diagnosis of SLOS was proven by serum 7DHC level. Anatomical abnormalities of ten embryologically separate organs (brain, oral region, limbs, eye, heart, kidney, liver, lung, bowel, and genitals) have been scored (5). On the basis of the clinical severity scores, patients were assigned to three groups: (i) patients with mild SLOS $(n=3$, clinical severity score $<20)$, (ii) typical SLOS ( $n=7$, clinical severity score $20-50)$ and (iii) severe SLOS $(n=1$, clinical severity score $>50$ ). After setting up the diagnosis, all patients received cholesterol supplementation (Cholesterol Module, $50-250 \mathrm{mg} / \mathrm{kg} / \mathrm{d}$, Nutricia; no 18.012), which was complemented with simvastatin in nine patients (dosage $0.2-0.4 \mathrm{mg} / \mathrm{kg} / \mathrm{d}$ ). None of the studied children received dietary and antioxidant supplements and were free of clinically significant infectious diseases. All parents provided written informed consent. The study protocol was approved by the Ethical Committee of University of Debrecen and the study was carried out in accordance with the Helsinki Declaration.

\section{Sample Collection and Laboratory Measurements}

Venous blood samples were taken after an overnight fasting and sera were separated immediately. Routine laboratory parameters were determined from fresh sera with Cobas c501 analyzer (Roche, Mannheim, Germany). Total cholesterol levels were measured by using enzymatic, colorimetric tests (cholesteroloxidase, p-aminophenazoneCHOD-PAP). HDL-cholesterol (HDL-C) levels were determined by a homogenous, enzymatic, colorimetric assay (Roche HDL-C plus third generation). The rapid determination of serum 7DHC was performed by an UV spectrophotometric method as described previously (8). Sera were kept frozen at $-70{ }^{\circ} \mathrm{C}$ for subsequent LDL and HDL subfraction analysis and PON1 activity measurements.

\section{LDL Subfraction Analysis}

LDL subfractions were detected by polyacrylamide gel electrophoresis using the Lipoprint System (Quantimetrix, Redondo Beach, CA) according to the manufacturer's instructions. Briefly, $25 \mu \mathrm{l}$ of serum samples were added to polyacrylamide gel tubes along with $200 \mu \mathrm{l}$ loading gel solution containing Sudan Black as lipophilic dye. After 30 min of photopolymerization at room temperature, electrophoresis was performed for $1 \mathrm{~h}$ with a $3 \mathrm{~mA} / g e l$ tube.

Lipoprotein fractions (bands) were identified after electrophoresis by their mobility (Rf) using VLDL as the starting reference point (Rf 0.0) and HDL as the ending reference point (Rf 1.0). In between, IDL subfractions (Midband $\mathrm{A}, \mathrm{B}$, and $\mathrm{C}$ ) and LDL subfractions were distributed from LDL1 to LDL7 (Rf 0.32, 0.38, 0.45, 0.51, 0.56, 0.6, and 0.64 , respectively). LDL1 and LDL2 bands corresponded to the larger, buoyant LDL subclasses (designed as normal "Pattern A"), whereas bands from LDL3 to LDL7 represented the predominantly smaller, denser LDL subclasses (designed as abnormal "Pattern B"). Percentages of the area under the curve (AUC\%) were calculated with Lipoware computer software (Quantimetrix). Proportion of large LDL (large LDL\%) was defined as the sum of the percentage of LDL1 and LDL2, whereas proportion of small LDL (small LDL\%) was defined as the sum of LDL3 to LDL7. Cholesterol concentrations of each lipoprotein bands were determined by multiplying the relative AUC of subfractions by total cholesterol concentration of the sample. LDL-cholesterol (LDL-C) is calculated by an automatic way using the Lipoware software as the sum of the cholesterol concentrations of all the LDL subfractions plus the Midband A, B, and C. Mean LDL size $(\mathrm{nm})$ was also calculated by the Lipoware software.

\section{HDL Subfraction Analysis}

HDL subfractions were also detected by polyacrylamide gelelectrophoresis with the Lipoprint System (Quantimetrix). $25 \mu$ of serum samples were added to polyacrylamide gel tubes along with $300 \mu \mathrm{l}$ loading gel solution containing Sudan Black as lipophilic dye. After 30 min of photopolymerization at room temperature, electrophoresis was performed for $50 \mathrm{~min}$ with a $3 \mathrm{~mA} / \mathrm{gel}$ tube.

Stained HDL subfractions (bands) were identified by their mobility after electrophoresis. The LDL/VLDL band was the starting reference point ( $R f 0.0$ ) and albumin was the ending reference point ( $\mathrm{Rf}$ 1.0). AUC\% were calculated with the Lipoware computer software (Quantimetrix). Up to $10 \mathrm{HDL}$ subfractions, distributed between the LDL/VLDL and albumin bands were grouped into three major classes: large (HDL1 to HDL3), intermediate (HDL4 to HDL7) and small (HDL8 to HDL10) HDL subfractions.

\section{Human Paraoxonase-1 Measurements}

PON1 paraoxonase activity was analyzed on a microtiter plate by a kinetic, semiautomated method utilizing paraoxon (O,O-diethylO-p-nitrophenyl-phosphate, Sigma Aldrich, Hungary) as a substrate. PON1 arylesterase activity was assayed with a phenylacetate substrate (Sigma Aldrich, Hungary) and the hydrolysis of phenylacetate was monitored at $270 \mathrm{~nm} \mathrm{(36).}$

\section{Statistical Analyses}

Statistical analysis was performed by STATISTICA software (ver 8.0; StatSoft, Tulsa, OK). The normality of data distribution was tested by Kolmogorov-Smirnov test. Data are presented by descriptive analysis (mean \pm SD in case of normal distribution, or median (lower quartile to upper quartile) in case of non-normal distribution). Comparisons between groups were performed by Student's paired $t$-test in case of normally distributed variables and by Mann-Whitney $U$-test in case of variables with non-normal distribution. Correlations between continuous variables were assessed by linear regression analysis using Pearson's test. Results were considered significant at the level of $P<0.05$.

\section{STATEMENT OF FINANCIAL SUPPORT}

The work is supported by a grant from the Hungarian Scientific Research Fund (OTKA 84196) and by the TÁMOP-4.2.2.A-11/1/KONV-2012-0031 project (University of Debrecen, Debrecen, Hungary). The TÁMOP project is cofinanced by the European Union and the European Social Fund.

Disclosure: There is no conflict of interest to disclose. 


\section{REFERENCES}

1. Orth M, Bellosta S. Cholesterol: its regulation and role in central nervous system disorders. Cholesterol 2012;2012:292598.

2. Schroepfer GJ Jr. Sterol biosynthesis. Annu Rev Biochem 1981;50:585-621.

3. Ryan AK, Bartlett K, Clayton P, et al. Smith-Lemli-Opitz syndrome: a variable clinical and biochemical phenotype. J Med Genet 1998;35:558-65.

4. DeBarber AE, Eroglu Y, Merkens LS, Pappu AS, Steiner RD. Smith-LemliOpitz syndrome. Expert Rev Mol Med 2011;13:e24.

5. Kelley RI, Hennekam RC. The Smith-Lemli-Opitz syndrome. J Med Genet 2000;37:321-35.

6. Tint GS, Irons M, Elias ER, et al. Defective cholesterol biosynthesis associated with the Smith-Lemli-Opitz syndrome. N Engl J Med 1994;330:107-13.

7. Tint GS, Salen G, Batta AK, et al. Correlation of severity and outcome with plasma sterol levels in variants of the Smith-Lemli-Opitz syndrome. J Pediatr 1995;127:82-7.

8. Oláh AV, Szabó GP, Varga J, et al. Relation between biomarkers and clinical severity in patients with Smith-Lemli-Opitz syndrome. Eur J Pediatr 2013;172:623-30.

9. Jira PE, Wevers RA, de Jong J, et al. Simvastatin. A new therapeutic approach for Smith-Lemli-Opitz syndrome. J Lipid Res 2000;41:1339-46.

10. Szabó GP, Oláh AV, Kozak L, et al. A patient with Smith-Lemli-Opitz syndrome: novel mutation of the DHCR7 gene and effects of therapy with simvastatin and cholesterol supplement. Eur J Pediatr 2010;169:121-3.

11. Fliesler SJ. Antioxidants: The Missing Key to Improved Therapeutic Intervention in Smith-Lemli-Opitz Syndrome? Hereditary Genet 2013;2:119.

12. Svoboda MD, Christie JM, Eroglu Y, Freeman KA, Steiner RD. Treatment of Smith-Lemli-Opitz syndrome and other sterol disorders. Am J Med Genet C Semin Med Genet 2012;160C:285-94.

13. Korade Z, Xu L, Mirnics K, Porter NA. Lipid biomarkers of oxidative stress in a genetic mouse model of Smith-Lemli-Opitz syndrome. J Inherit Metab Dis 2013;36:113-22.

14. Liu W, Xu L, Lamberson CR, et al. Assays of plasma dehydrocholesteryl esters and oxysterols from Smith-Lemli-Opitz syndrome patients. J Lipid Res 2013;54:244-53.

15. Mertens A, Holvoet P. Oxidized LDL and HDL: antagonists in atherothrombosis. FASEB J 2001;15:2073-84.

16. Mackness B, Mackness MI, Arrol S, Turkie W, Durrington PN. Effect of the human serum paraoxonase 55 and 192 genetic polymorphisms on the protection by high density lipoprotein against low density lipoprotein oxidative modification. FEBS Lett 1998;423:57-60.

17. Macharia M, Hassan MS, Blackhurst D, Erasmus RT, Matsha TE. The growing importance of PON1 in cardiovascular health: a review. J Cardiovasc Med (Hagerstown) 2012;13:443-53.

18. Koncsos P, Seres I, Harangi M, et al. Human paraoxonase- 1 activity in childhood obesity and its relation to leptin and adiponectin levels. Pediatr Res 2010;67:309-13.

19. Kiss E, Seres I, Tarr T, Kocsis Z, Szegedi G, Paragh G. Reduced paraoxonase1 activity is a risk for atherosclerosis in patients with systemic lupus erythematosus. Ann N Y Acad Sci 2007;1108:83-91.
20. Szántó A, Harangi M, Seres I, Paragh G, Zeher M. Decreased human paraoxonase-1 activity in patients with Sjögren's syndrome. Int Immunol 2010;22:605-9.

21. Korade Z, Xu L, Shelton R, Porter NA. Biological activities of 7-dehydrocholesterol-derived oxysterols: implications for Smith-Lemli-Opitz syndrome. J Lipid Res 2010;51:3259-69.

22. Xu L, Korade Z, Rosado DA Jr, Liu W, Lamberson CR, Porter NA. An oxysterol biomarker for 7-dehydrocholesterol oxidation in cell/ mouse models for Smith-Lemli-Opitz syndrome. J Lipid Res 2011;52: 1222-33.

23. Merkens LS, Connor WE, Linck LM, Lin DS, Flavell DP, Steiner RD. Effects of dietary cholesterol on plasma lipoproteins in Smith-Lemli-Opitz syndrome. Pediatr Res 2004;56:726-32.

24. Behúlová D, Bzdúch V, Skodová J, et al. Serum lipids and apolipoproteins in children with the Smith-Lemli-Opitz syndrome. J Inherit Metab Dis 2000;23:413-5.

25. Krauss RM. Lipoprotein subfractions and cardiovascular disease risk. Curr Opin Lipidol 2010;21:305-11.

26. Kaitosaari T, Simell O, Viikari J, et al. Tracking and determinants of LDL particle size in healthy children from 7 to 11 years of age: the STRIP Study. Eur J Pediatr 2009;168:531-9.

27. Kaji H. High-density lipoproteins and the immune system. J Lipids 2013;2013:684903.

28. Kontush A, Chapman MJ. Antiatherogenic small, dense HDL-guardian angel of the arterial wall? Nat Clin Pract Cardiovasc Med 2006;3: $144-53$.

29. Schiavon R, Battaglia P, De Fanti E, et al. HDL3-related decreased serum paraoxonase (PON) activity in uremic patients: comparison with the PON1 allele polymorphism. Clin Chim Acta 2002;324:39-44.

30. Kontush A, Chantepie S, Chapman MJ. Small, dense HDL particles exert potent protection of atherogenic LDL against oxidative stress. Arterioscler Thromb Vasc Biol 2003;23:1881-8.

31. Camps J, Marsillach J, Joven J. The paraoxonases: role in human diseases and methodological difficulties in measurement. Crit Rev Clin Lab Sci 2009;46:83-106.

32. Furlong CE, Holland N, Richter RJ, Bradman A, Ho A, Eskenazi B. PON1 status of farmworker mothers and children as a predictor of organophosphate sensitivity. Pharmacogenet Genomics 2006;16:183-90.

33. Deakin SP, James RW. Genetic and environmental factors modulating serum concentrations and activities of the antioxidant enzyme paraoxonase-1. Clin Sci 2004;107:435-47.

34. Bergmeier C, Siekmeier R, Gross W. Distribution spectrum of paraoxonase activity in HDL fractions. Clin Chem 2004;50:2309-15.

35. Korade Z, Xu L, Harrison FE, et al. Antioxidant supplementation ameliorates molecular deficits in Smith-Lemli-Opitz syndrome. Biol Psychiatry 2014;75:215-22.

36. Fülöp P, Seres I, Lorincz H, Harangi M, Somodi S, Paragh G. Association of chemerin with oxidative stress, inflammation and classical adipokines in non-diabetic obese patients. J Cell Mol Med 2014;18:1313-20. 